$63^{\text {ème }}$ Congrès de la SFCO, 03002 (2015)

DOI:10.1051/sfco/20156303002

(C) Owned by the authors, published by EDP Sciences, 2015

\title{
Découverte fortuite d'un méningiome ayant pour manifestation clinique une névralgie trigéminale symptomatique
}

\author{
Malthièry $\mathrm{E}^{*}$, Kimakhe $\mathrm{S}^{*}$, Torres $\mathrm{J}-\mathrm{H}^{* *}$ \\ * Service d'Odontologie, CHU Nantes, 1 place Alexis Ricordeau, 44093 Nantes \\ ** Service d'Odontologie, CHRU Montpellier, 545 avenue du Pr. Jean-Louis Viala, 34295 Montpellier
}

Les douleurs oro-faciales sont souvent sévères et handicapantes d'un point de vue social et psychologique. La sphère oro-faciale est caractérisée par une richesse nerveuse ayant pour conséquence une expression multiple des douleurs oro-faciales (Tomoyasu et al. 2014). Leur diagnostic est donc parfois malaisé. Les névralgies essentielles du nerf trijumeau se manifestent généralement d’une manière stéréotypée. En revanche, les autres névralgies trigéminales, dites symptomatiques, plus rares, se manifestent parfois par des tableaux cliniques atypiques et demandent des explorations plus approfondies (Shulev et al. 2011).

Une patiente de 58 ans a consulté pour des douleurs à point de départ buccal. Celles-ci étaient décrites comme des décharges électriques régulières avec des phases de rémission de plus en plus réduites, intéressant strictement le côté gauche mandibulaire postérieur. Ces douleurs débutaient au corps mandibulaire, empruntaient la branche pour finalement irradier vers la tempe gauche (grande aile du sphénoïde et partie antérieure du temporal). La patiente, édentée totale, portait des prothèses amovibles depuis 13 ans. L'examen clinique n’a trouvé aucune lésion inflammatoire ni traumatique sur les muqueuses buccales, aucune douleur articulaire, ni otalgie, ni myalgie. Il n'a objectivé aucune zone hyperalgique ni aucun déficit sensitif. La radiographie panoramique dentaire n’a montré aucune image osseuse susceptible d'expliquer la manifestation algique.

Devant l'absence d'éléments en faveur d'une origine locale, une étiologie neurologique centrale a été évoquée et la patiente a été adressée au centre d'évaluation et de traitement de la douleur où un scanner a montré la présence d'une masse cérébrale radioclaire gauche et orienté le diagnostic vers un méningiome malin. Cette lésion, étendue de la région ponto-cérébelleuse jusqu'au gyrus central était, dans sa partie inférieure, en contact avec le nerf trijumeau gauche. Le rapport étroit de cette tumeur avec l'artère sylvienne homolatérale contre-indiquait une intervention chirurgicale en première intention, le risque hémorragique étant trop élevé. En réunion de concertation pluridisciplinaire (RCP), il a été prévu tout d'abord une radiothérapie conformationnelle afin de réduire la masse tumorale puis une éventuelle chirurgie. Le traitement n'a pas pu être conduit à son terme, la patiente ayant succombé quelques semaines plus tard suite à une hémorragie cérébrale, présumée en lien avec cette tumeur.

Ce cas clinique nous rappelle l'intérêt d'être à la fois vigilants et réactifs sur ce type de névralgie symptomatique à point de départ non buccal, le pronostic vital pouvant être engagé. Il met aussi en lumière le fait que le praticien doit avoir toujours présent à l'esprit une démarche clinique ouverte et multidisciplinaire. 
$63^{\text {ème }}$ Congrès de la SFCO

Nom et adresse du conférencier

Eve MALTHIÈRY

Service d'Odontologie, CHU Nantes

1 place Alexis Ricordeau

44093 Nantes (France)

eve.malthiery@univ-nantes.fr 